\title{
Research Article \\ Optimal On-Off Attitude Control for the Brazilian Multimission Platform Satellite
}

\author{
Gilberto Arantes Jr., ${ }^{1}$ Luiz S. Martins-Filho, ${ }^{2}$ \\ and Adrielle C. Santana ${ }^{3}$ \\ ${ }^{1}$ Center for Applied Space Technology and Microgravity, (ZARM) Am Fallturm, University of Bremen, \\ 28359 Bremen, Germany \\ ${ }^{2}$ Center of Engineering, Modelling and Applied Social Sciences, Federal University of ABC, \\ Rua Catequese 242, 09090-400 Santo André, SP, Brazil \\ ${ }^{3}$ Graduate Program on Information Engineering, Federal University of ABC, Rua Catequese 242, \\ 09090-400 Santo André, SP, Brazil
}

Correspondence should be addressed to Luiz S. Martins-Filho, luizsmf@gmail.com

Received 24 June 2009; Accepted 13 August 2009

Recommended by Maria Zanardi

This work deals with the analysis and design of a reaction thruster attitude control for the Brazilian Multimission platform satellite. The three-axis attitude control systems are activated in pulse mode. Consequently, a modulation of the torque command is compelling in order to avoid high nonlinear control action. This work considers the Pulse-Width Pulse-Frequency (PWPF) modulator which is composed of a Schmidt trigger, a first-order filter, and a feedback loop. PWPF modulator holds several advantages over classical bang-bang controllers such as close to linear operation, high accuracy, and reduced propellant consumption. The Linear Gaussian Quadratic (LQG) technique is used to synthesize the control law during stabilization mode and the modulator is used to modulate the continuous control signal to discrete one. Numerical simulations are used to analyze the performance of the attitude control. The LQG/PWPF approach achieves good stabilization-mode requirements as disturbances rejection and regulation performance.

Copyright (C) 2009 Gilberto Arantes Jr et al. This is an open access article distributed under the Creative Commons Attribution License, which permits unrestricted use, distribution, and reproduction in any medium, provided the original work is properly cited.

\section{Introduction}

One of the intentions of this work is to support the ongoing Brazilian multimission platform (MMP) satellite project [1]. The project takes into consideration a special platform satellite which can supply multimissions capabilities supporting different payloads to lift up on the platform. Applications including Earth observation, communication, scientific experiments, and surveillance are few examples of suitable use of the MMP satellite. The MMP adopted pulse or on-off reaction thruster for attitude maneuvers, therefore, modulating continuous command signal to an on-off signal is a required task. Selecting the properly method to 
modulate the control command signal is a key assignment. The aim of this work is to provide smoother control for improved pointing requirements with less thruster activation or propellant consumption. The fuel is a deciding factor of the lifetime of the spacecraft and reduced propellant consumption is highly required, specially, regarding a multimission spacecraft wherein different payloads are being considered.

In this paper a pulse-width pulse-frequency (PWPF) modulator is considered as a feasible option for the MMP reaction thruster modulation due to advantages over other types of pulse modulators as bang-bang controllers which has excessive thruster actuation $[2,3]$. The PWPF modulator translates the continuous commanded control/torque signal to an onoff signal. Its behavior is a quasilinear mode which is possible by modulating the width of the activated reaction pulse proportionally to the level of the torque command input (pulsewidth) and also the distance between the pulses (pulse-frequency). A PWPF modulator is composed of a Schmidt trigger, a lag network filter, and a feedback loop. The PWPF design requires iterative tuning of lag filter and Schmidt trigger. The optimal parameters achievement is based on the static (test signals) and dynamic (feedback signals) simulation results. The optimality is in respect to either the number of firings or spent fuel. The work in [3-5] provides good guidelines for the PWPF tuning task.

The PWPF is synthesized with a Linear Quadratic Gaussian (LQG) controller which is designed for the MIMO attitude system. The LQG controller, refered to as $\mathfrak{t}_{2}$, allows a tradeoff between regulation performance and control effort. In order to reduce the control effort or fuel consumption, an iteratively searching of the trade-off can be carried out. Nevertheless the controller has to attempt all the involved requirements and specifications. A previous study of the LQG approach applied to the MMP satellite is presented in [6]. The reaction attitude control system is applied to the stabilization mode of the MMP. The paper is divided into 5 sections. Section 2 presents the nonlinear model of the satellite, assumed a rigid body, its linearization around the operation point, and the developed virtual reality model of the satellite for visualization purposes. Section 3 presents a brief description of the PWPF modulator and design of the LQG controller, which includes the description of the LQG controller and provides the tuning parameters range for the PWPF modulator. Section 4 presents the numerical simulation for the reaction thruster attitude control system during the stabilization mode. Regulation, filtering, and disturbance rejection are investigated and discussed. Conclusions are presented in Section 5 based on the obtained results.

\section{Problem Formulation}

In this section we describe the mathematical model of the attitude motion, including kinematics, dynamics, and the linerization of the satellite model around LHLV reference frame. Based on that linear model the LQG controller is designed for the stabilization mode.

\subsection{Satellite Attitude Model}

The attitude of the satellite will be defined in this work by the orientation of the body frame $(x, y, z)$ (coincident with the three principal axes of inertia) with respect to the orbital reference frame $\left(x_{r}, y_{r}, z_{r}\right)$, also known as Local-Vertical-Local-Horizontal (LVLH) [7]. The origin of the orbit reference frame moves with the center of mass of the satellite in orbit. The $z_{r}$ axis points toward the center of mass of the Earth, $x_{r}$ axis is in the plane of the orbit, perpendicular to $z_{r}$, in the direction of the velocity of the spacecraft. The $y_{r}$ axis is normal to 


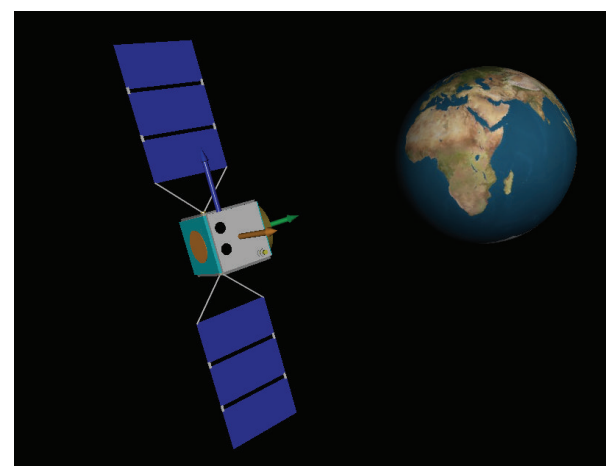

Figure 1: LVLH axis representation.

the local plane of the orbit, and completes a three-axis right-hand orthogonal system. Figure 1 illustrated the LVLH reference frame.

The attitude is represented by the direction cosine matrix $R$ between body frame and reference frame. During the stabilization mode only small angular variations are considered, in this case the Euler angles parametrization is an appropriate choice due to the guarantee of nonsingularity. Thus, by using Euler angles $(\phi, \theta, \psi)$ in an asymmetric sequence 3-2-1 (z-y-x) for describing a rotation matrix, one finds $[7,8]$

$$
R_{z y x}=\left[\begin{array}{ccc}
c \psi c \theta & s \psi c \theta & -s \theta \\
-c \phi s \psi+s \phi s \theta c \psi & c \phi c \psi+s \phi s \theta s \psi & s \phi c \theta \\
s \phi s \psi+c \phi s \theta c \psi & -s \phi c \psi+c \phi s \theta s \psi & c \phi c \theta
\end{array}\right] .
$$

For a rotating body the elements of the direction cosine matrix change with time, this change relative to any reference frame fixed in inertial space can be written as follows [9]:

$$
\dot{R}(t)=S\left(\omega_{i b}^{b}\right) R(t)
$$

where $\omega_{i b}^{b}=\left(\omega_{x}, \omega_{y}, \omega_{z}\right)^{T}$ is the angular velocity of the body frame relative to the inertial frame, expressed in the body frame, $S$ is the skew-symmetric operator given by

$$
S\left(\boldsymbol{\omega}_{i b}^{b}\right)=\left[\begin{array}{ccc}
0 & -\omega_{z} & \omega_{y} \\
\omega_{z} & 0 & -\omega_{x} \\
-\omega_{y} & \omega_{x} & 0
\end{array}\right]
$$

According to [10] the angular velocity can be expressed as function of the mean orbital motion $\left(\omega_{0}\right)$ and the derivatives $(\dot{\phi}, \dot{\theta}, \dot{\phi})$, thereafter the kinematics of the rigid body is 
expressed by

$$
\boldsymbol{\omega}_{i b}^{b}=\left[\begin{array}{ccc}
1 & 0 & -s \theta \\
0 & c \phi & s \phi c \theta \\
0 & -s \phi & c \phi c \theta
\end{array}\right]\left[\begin{array}{c}
\dot{\phi} \\
\dot{\theta} \\
\dot{\psi}
\end{array}\right]-\omega_{0}\left[\begin{array}{c}
c \theta s \psi \\
s \phi s \theta s \psi+c \phi c \psi \\
c \phi s \theta s \psi-s \phi c \psi
\end{array}\right]
$$

since large slewing maneuvers of the satellite are not considered, it is save to approximate $c \theta \approx 1, s \theta \approx \theta, \phi \psi \approx 0$. According to (2.4) for small Euler angles, the kinematics can be approximated as

$$
\boldsymbol{\omega}_{i b}^{b}=\left[\begin{array}{c}
\dot{\phi} \\
\dot{\theta} \\
\dot{\psi}
\end{array}\right]+\omega_{0}\left[\begin{array}{c}
-\psi \\
-1 \\
\phi
\end{array}\right]
$$

The dynamics of a satellite attitude, equipped with six one-sides thrusters is modelled by using the Euler equations. Furthermore, the attitude dynamic is written in the body frame, it yields

$$
\tau_{\text {ext }}=\left[\frac{d \mathbf{h}}{d t}\right]_{b}+\boldsymbol{\omega}_{i b}^{b} \times \mathbf{h}_{b}
$$

where $\mathbf{h}_{b}=J \boldsymbol{\omega}_{i b}^{b}$ is the momentum of the rigid body, $J$ is the satellite inertia matrix, and $\boldsymbol{\tau}_{\text {ext }}$ are the external torques acting in the system including perturbation and thruster actuation. Using $[d \mathbf{h} / d t]_{b}=J \dot{\boldsymbol{\omega}}_{i b^{\prime}}^{b}(2.6)$ becomes

$$
J \dot{\boldsymbol{\omega}}_{i b}^{b}+S\left(\boldsymbol{\omega}_{i b}^{b}\right) J \boldsymbol{\omega}_{i b}^{b}=\boldsymbol{\tau}_{d}^{b}+\boldsymbol{\tau}_{c}^{b}
$$

where $\boldsymbol{\tau}_{d}^{b}$ represents all the disturbance torques, for example, atmosphere drag, gravity gradient, and so on, and $\boldsymbol{\tau}_{c}^{b}$ represents the control torques used for controlling the attitude motion. The control torques about the body axes, $x, y$, and $z$ provide by the thrusters are $\boldsymbol{\tau}_{x}, \boldsymbol{\tau}_{y} \boldsymbol{\tau}_{z}$, respectively. The thruster reaction system is discussed in detail in the following section. The torque effect caused by the gravity gradient is taken into account and it is included in the linearization process. An asymmetric body subject to a gravitational field experience a torque tending to align the axis of the least moment of inertia with the field direction [8]. For small angle maneuvers, the model of the gravity gradient torque is approximated as $[8,9]$

$$
\boldsymbol{\tau}_{g}^{b}=3 \omega_{0}^{2}\left[\begin{array}{c}
\left(J_{z}-J_{y}\right) \phi \\
\left(J_{x}-J_{z}\right) \theta \\
0
\end{array}\right]
$$

Substituting (2.4) into (2.6) and adding the control and gradient gravity torque, we linearize the satellite attitude model. Moreover, the linearization is performed around the LHLV orbital 
frame, it is thus adopted for the stabilization mode. Afterwards the attitude model can be represented in the state space form $[6,10]$

$$
\begin{aligned}
& \dot{\mathbf{x}}=A \mathbf{x}+B \mathbf{u}, \\
& \mathbf{y}=C \mathbf{x}+D \mathbf{u},
\end{aligned}
$$

with states $\mathbf{x}=[\phi, \theta, \psi, \dot{\phi}, \dot{\theta}, \dot{\psi}]^{T}$, and inputs $\mathbf{u}=\left[\tau_{x}, \tau_{y}, \tau_{z}\right]^{T} . A$ is the state matrix, $B$ is the input matrix, $C$ is the output matrix, and $D$ is the direct transmission matrix. In the particular problem they are given by

$$
\begin{aligned}
& A=\left[\begin{array}{cccccc}
0 & 0 & 0 & 1 & 0 & 0 \\
0 & 0 & 0 & 0 & 1 & 0 \\
0 & 0 & 0 & 0 & 0 & 1 \\
\frac{4 \omega_{0}^{2}\left(J_{z}-J_{y}\right)}{J_{x}} & 0 & 0 & 0 & 0 \frac{\omega_{0}\left(J_{x}-J_{y}+J_{z}\right)}{J_{x}} \\
0 & \frac{3 \omega_{0}^{2}\left(J_{x}-J_{z}\right)}{J_{y}} & 0 & 0 & 0 & 0 \\
0 & 0 & \frac{\omega_{0}^{2}\left(J_{x}-J_{y}\right)}{J_{z}} \frac{\omega_{0}\left(J_{y}-J_{x}-J_{z}\right)}{J_{z}} & 0 & 0
\end{array}\right], \\
& B=\left[\begin{array}{ccc}
0 & 0 & 0 \\
0 & 0 & 0 \\
0 & 0 & 0 \\
\frac{l}{J_{x}} & 0 & 0 \\
0 & \frac{l}{J_{y}} & 0 \\
0 & 0 & \frac{l}{J_{z}}
\end{array}\right], \quad C=I_{6 \times 6}, \quad D=0_{6 \times 3} .
\end{aligned}
$$

It is worth to note that $x$ row and $z$ yaw axes belong to a multi-input and multi-output (MIMO) system $4 \times 2$ and the $y$ pitch axis could be dealt as a single input and single output system (SISO) by assuming a tachometry feedback control. Although the controller is project over the linear model, the nonlinear model is used in the simulations.

\subsection{Virtual Reality Model of the Spacecraft}

In this work a Virtual Reality (VR) model are developed as a visualization tool. The purpose is to visualize the simulations giving a fast and a visual feedback of the simulation models over time. The model is produced by using the virtual reality model language (VRML) format which includes a description of 3-dimensional scenes, sounds, internal actions, and WWW anchors. It enables us to view moving three-dimensional scenes driven by signals from the 


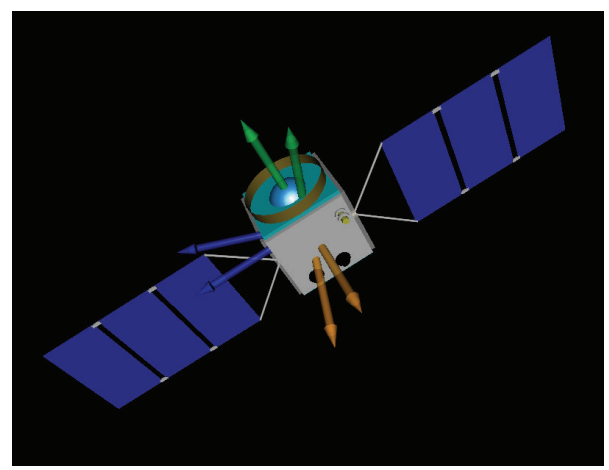

Figure 2: A graphical interface in VRML for visualization.

dynamic model, that is, attitude dynamics. The VR model was created with the use of VRealm builder tool, a more detailed description can be found in [11]. Figure 2 shows the basic structure representation of the spacecraft's bus. The payload is not illustrated.

\section{Thruster Attitude Control System}

In this section the controller design based on the Linear Quadratic Gaussian (LQG) technique is briefly described, afterwards the PWPF modulator is presented in details.

\subsection{LQG Controller Design}

The Linear Quadratic Gaussian (LQG) or $\mathfrak{l}_{2}$ control consist of a technique for designing optimal controllers. The approach is based on the search of the tradeoff between regulation performance of the states and control effort [12]. The referred optimality is expressed by a quadratic cost function and allows the designer to shape the principal gains of the return ration, at either the input or the output of the plant, to achieve required performance or robustness specifications. Moreover the method is easily designed for Multi-Input MultiOutput (MIMO) systems. The controller design takes into account disturbances in the plant and measurement noise from the sensors. Formally, the LQG approach addresses the problem where we consider a linear system model perturbed by disturbances $\mathbf{w}$, and measurements of the sensor corrupted by noise $\boldsymbol{v}$ which includes also the effects of the disturbances by measurement environment. The state-space model representation of the linear or linearized system with the addition of the disturbance effects can be mathematically expressed by

$$
\begin{aligned}
\dot{\mathbf{x}} & =A \mathbf{x}+B \mathbf{u}+G \mathbf{w}, \\
\mathbf{y}_{v} & =C \mathbf{x}+D \mathbf{u}+\boldsymbol{v},
\end{aligned}
$$

in our problem $A, B, C$, and $D$ are given by (2.10). The matrix $G$ is the disturbance balance matrix. The disturbance and measurements noises are assumed both white noises. The principle of the LQG is combine the linear quadratic regulator (LQR) and the linear-quadratic estimator (LQE), that is, a steady-state Kalman filter. The separation principle guarantees that those can be design and computed independently [13]. 


\subsubsection{LQR Problem}

The solution for the optimal state feedback controller is obtained by solving the LQR problem. Namely the LQR optimal controller automatically ensures a stable close-loop system, and achieves guarantee levels of stability and robustness for minimal phase systems, for example, multivariable margins of phase and gain. The LQR approach gives the optimal controller gain, denoted by $K$, with linear control law:

$$
\mathbf{u}=-K \mathbf{x},
$$

which minimizes the quadratic cost function, given by

$$
J_{L Q R}=\int_{0}^{\infty}\left(\mathbf{x}^{T} Q \mathbf{x}+\mathbf{u}^{T} R \mathbf{u}\right) d t
$$

where $Q$ is positive definite, and $R$ is semipositive definite, these are weighting or tuning matrices that define the trade-off between regulation performance and control efforts. The first term in (3.3) corresponds to the energy of the controlled output $(\mathbf{y}=\mathbf{x})$ and the second term corresponds to the energy of the control signal. The gain matrix $K$ for the optimization problem is obtained by solving the algebraic matrix Riccati equation:

$$
A^{T} P+P A-P B R^{-1} B^{T} P+Q=0 .
$$

The optimal control gain is then obtained by

$$
K=R^{-1} B^{T} P .
$$

The close-loop dynamics model is obtained by substituting (3.5) into (3.1), and taking $\mathbf{w}=$ $\mathbf{v}=0$, as follows

$$
\dot{\mathbf{x}}=(A-B K) \mathbf{x},
$$

which corresponds to an asymptotically stable system.

In order to adopt the LQR formulation the whole state $\mathbf{x}$ of the process has to be measurable. In this case it is necessary to estimate the absent states, so the estimated states are denoted by $\widehat{\mathbf{x}}$. Notice that the output matrix in our case is $C=I_{6 \times 6}$, it means that the whole state is measurable. Physically, the angular rates are obtained from the gyros and the attitude/orientation from the solar sensor. Nevertheless, because of the presence of noise, an estimation is advice in order to produce better and reliable information about the real states. The estimation is performed by employing the steady-state Kalman filter. 


\subsubsection{Kalman Filter Design}

The Kalman filter is used to obtain the estimated state $\widehat{\mathbf{x}}$. The filter equation in view of the attitude model is given by

$$
\dot{\hat{\mathbf{x}}}=A \widehat{\mathbf{x}}+B \mathbf{u}+L\left(\mathbf{y}_{v}-C \widehat{\mathbf{x}}\right)
$$

where $L$ is the Kalman filter gain. The optimal gain $L$ minimizes the covariance of the error $E$ between real $\mathbf{x}$ and estimated $\widehat{\mathbf{x}}$ states, by defining the state estimation error as $\mathbf{e}:=\widehat{\mathbf{x}}-\mathbf{x}$, the cost function is given by [13]

$$
J_{L Q E}=\lim _{t \rightarrow \infty} E\left\{\mathbf{e} \cdot \mathbf{e}^{T}\right\}
$$

We assume that the disturbances affecting the process $\mathbf{w}$ and $\mathbf{v}$ are zero-mean Gaussian whitenoise process with covariances $Q_{e}=E\left(\mathbf{w} \mathbf{w}^{T}\right)$ and $R_{e}=E\left(\mathbf{v v}^{T}\right)$, respectively. The process and measurement noises are uncorrelated from each other. The gain $L$ is obtained solving the algebraic matrix Riccati equation:

$$
A^{T} S+S A-S C R_{e}^{-1} C^{T} S+Q_{e}=0 .
$$

The optimal estimator gain is then obtained by

$$
L=R_{e}^{-1} C^{T} S
$$

and the error dynamics is given by

$$
\dot{\mathbf{e}}=(A-L C) \mathbf{e},
$$

where $A-L C$ is asymptotically stable. From (3.6) and (3.11) the open-loop transfer function for the LQG controller is found as follows:

$$
K_{l q g}(s) G(s)=K(s I-A+B K+L C)^{-1} L \Phi(s),
$$

where $G(s)=\Phi(s)=C(s I-A)^{-1} B$ is the transfer function of the attitude model, in this case $G_{S}$ is a matrix of transfer functions.

\subsection{Pulse-Width Pulse-Frequency Modulator}

The control signals from the LQG controller are of continuous type. However, pulse thruster devices can provide only on-off signals generating nonlinear control action. Nonetheless, those can be used in a quasilinear mode by modulating the width of the activate reaction pulse proportionally to the level of the torque command input. This is known as pulse-width modulation $(\mathrm{PW})$. In the pulse-width pulse-frequency (PWPF) modulation the distance between the pulses is also modulated. Its basic structure is shown in Figure 3. 


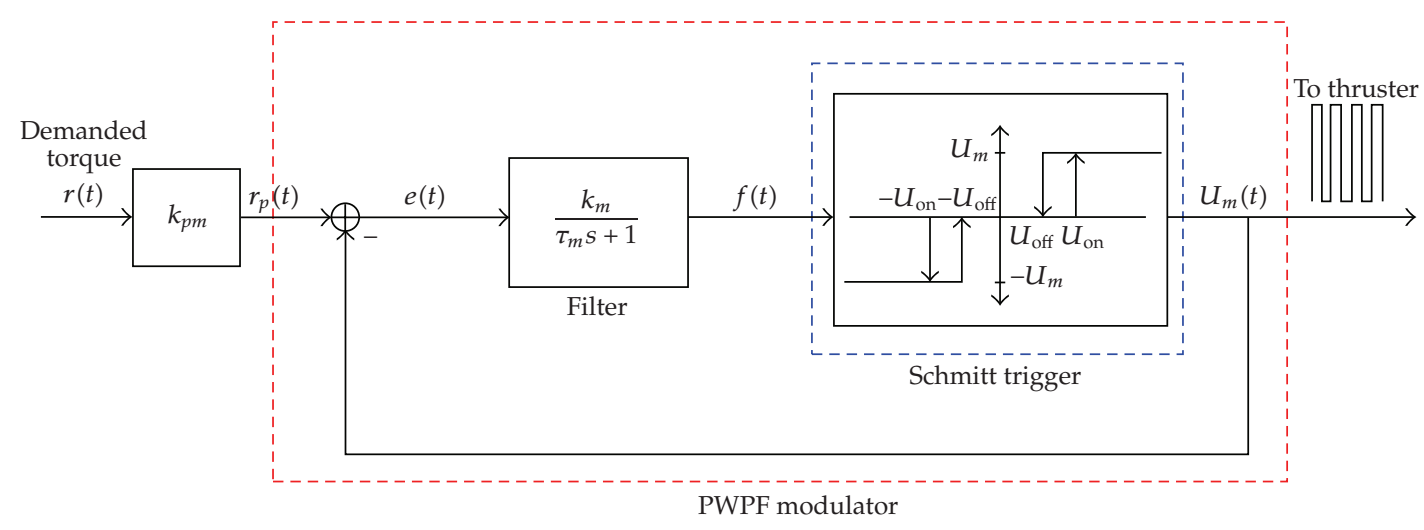

Figure 3: Pulse-width pulse-frequency (PWPF) modulator.

The modulator includes a Schmitt trigger which is a relay with dead zone and hysteresis, it includes also a first-order-filter, lag network type, and a negative feedback loop. When a positive input to the Schmitt trigger is greater than $U_{\text {on }}$, the trigger input is $U_{m}$. If the input falls below $U_{\text {off }}$ the trigger output is 0 . This response is also reflected for negative inputs in case of two side-thrusters or those thruster that produce negative torques (clockwise direction). The error signal $e(t)$ is the difference between the Schmitt trigger output $U_{\mathrm{on}}$ and the system input $r(t)$. The error is fed into the filter whose output signal $f(t)$ and it feeds the Schmitt trigger. The parameters of interest for designing the PWPF are: the filter coefficients $k_{m}$ and $\tau_{m}$, the Schmitt trigger parameters $U_{\text {on }}, U_{\text {off }}$, it defines the hysteresis as $h=U_{\text {on }}-U_{\text {off }}$, and the maximal/minimal $\pm U_{m}$. The PWPF modulator can incorporate an additional gain $k_{p m}$ which will be considered separately from the control gain.

In the case of a constant input, the PWPF modulator drives the thruster valve with on-off pulse sequence having a nearly linear duty cycle with input amplitude. It is worth to note that the modulator has a behavior independent of the system in which it is used [3]. The static characteristics of the continuous time modulator for a constant input $C$ are presented as follows:

(i) on-time

$$
T_{\mathrm{on}}=P W=-\tau_{m} \ln \left(1+\frac{h}{k_{m}\left(C-U_{m}\right)-U_{\mathrm{on}}}\right)
$$

(ii) off-time

$$
T_{\text {off }}=-\tau_{m} \ln \left(1-\frac{h}{k_{m} C-\left(U_{\mathrm{on}}-h\right)}\right),
$$

(iii) modulator frequency

$$
f=\frac{1}{T_{\text {on }}+T_{\text {off }}}
$$


Table 1: Recommended range for the PWPF parameters.

\begin{tabular}{lccc}
\hline & Static analysis & Dynamic analysis & Recommended \\
\hline$k_{m}$ & $2<k_{m}<7$ & $N / A$ & $2<k_{m}<7$ \\
$\tau_{m}$ & $0.1<\tau_{m}<1$ & $0.1<\tau_{m}<0.5$ & $0.1<\tau_{m}<.5$ \\
$U_{\mathrm{on}}$ & $U_{\mathrm{on}}>0.3$ & $N / A$ & $U_{\mathrm{on}}>0.3$ \\
$h$ & $h>0.2 U_{\mathrm{on}}$ & $N / A$ & $h>0.2 U_{\mathrm{on}}$ \\
\hline$k_{p m}$ & $N / A$ & $k_{p m} \geq 20$ & $k_{p m} \geq 20$ \\
\hline
\end{tabular}

(iv) duty cycle

$$
D C=\left[\frac{\ln [1+a /(1-x)]}{1+\ln [1+a / x]}\right]^{-1}
$$

(v) minimum pulse-width (PW)

$$
\Delta=-\tau_{m} \ln \left[1-\frac{h}{k_{m} U_{m}}\right]
$$

where the following internal parameters are also defined: dead zone $C_{d}=U_{\mathrm{on}} / k_{m}$, saturation level $C_{s}=U_{m}+\left(U_{\mathrm{on}}-h\right) / k_{m}$, normalized hysteresis width $a=h / k_{m}\left(C_{s}-C_{d}\right)$, and normalized input $x=\left(C-C_{d}\right) /\left(C_{s}-C_{d}\right)$.

In order to determine the range of parameters for the PWPF modulator, static and dynamic analyses are carried out. The static analysis involves test input signals, for example, step, ramp, and sinusoidal signals. The dynamic analysis uses plant and controller. Afterwards the choice is based upon the number of firings and level of fuel consumption results. The number of firings gives an indication of the life-time of the thrusters. Table 1 presents the obtained results for the particular problem.

\subsection{Specifications and Tuning Schemes}

The specification of the requirements for the attitude control system are determined by the capabilities of the MMP satellite to attempt some desired nominal performance for the linked payload. Considering the stabilization mode the following specifications are given in terms of time and frequency domain:

(i) steady state error less than $0.5^{\circ}$ degrees for each axis;

(ii) overshoot less than $40 \%$;

(iii) short rise time or fast response against disturbances;

(iv) stability margins gain $\mathrm{GM} \geq 6 \mathrm{db}$ and $\mathrm{PM} \geq 60^{\circ}$ for each channel.

For the control design, it is necessary to check the limitations and constraints imposed by the plant. In this sense the optimality of the LQG only holds for the following assumptions: the matrix $\left[\begin{array}{ll}A & B\end{array}\right]$ must be stabilizable and $\left[\begin{array}{ll}A & C\end{array}\right]$ must be detectable. In the case of the attitude model, both conditions are satisfied. The next step is to design a controller which achieves the required system performance. During the stabilization mode, it is desired 
attenuation of the effects of disturbances acting on the satellite and accomplishment of regulation to maintain the satellite in the required attitude. Moreover the output has to be insensitive to measurements errors. Unfortunately there is an unavoidable tradeoff between attenuation disturbances and filtering out measurement error. This tradeoff has to be kept in mind during the design of the controller. In the case of attitude model, the disturbances acting in the system belong to the spectrum at low frequencies, note that the regulation signals belongs also to spectrum at low frequencies. On the other hand, the measurement noises and unmodeled system terms are concentrated at high frequencies. In order to fulfill the specifications, tuning of LQG gains and PWPF gains have to be careful performed. The nature of the tuning is an iterative process which turns out less arduous with the use of a computational tool, in this work the Matlab package is used. In the following, the obtained weights for LQG controller and PWPF modulator are presented.

\subsubsection{LQR Tuning}

The first choice for the tuning matrices $Q$ and $R$ is taken from the Bryson's rule, selecting $Q$ and $R$ diagonal matrices with the form

$$
\begin{aligned}
& Q_{i i}=\frac{1}{\text { maximum acceptable value of } x_{i}^{2}} \quad i \in\{1,2, \ldots, n\}, \\
& R_{i i}=\frac{1}{\text { maximum acceptable value of } u_{j}^{2}} \quad j \in\{1,2, \ldots, m\},
\end{aligned}
$$

where $x_{i}$ and $u_{j}$ are the states input signals boundaries, respectively. The rule is used to keep the states and inputs below some boundaries. It is advised to avoid large control signals which from the engineering point of view are unacceptable. On the other hand, the controller has to fulfill all the system specifications and the LQR formulation does not directly allow one to achieve standard control system specifications. Nevertheless those can be achieved by iteration over the values of the weights of $Q$ and $R$ in the cost function till it arrives at satisfactory controller. For the proposed reaction attitude control system the boundaries for the states are kept $\pm 5^{\circ}$ in attitude $(\phi, \theta, \psi)$, and \pm 1 degree per second for the rates. The boundary for the input signals are 1 Newton meter. The result weighting matrices for the controller which achieved satisfactory controller are

$$
Q=Q_{i i}, \quad R=1 \times 10^{-1} \cdot R_{i i}
$$

The control tuning matrices $R$ and $Q$ were obtained through iterative process following expectable requirements, for example, allowed (non-saturation) control effort and reasonable stabilization time.

\subsubsection{Filter Tuning}

The tuning weight matrices $R_{e}$ and $Q_{e}$ for the Kalman filter are obtained considering $R_{e}$ large compared to $Q_{e}$. It corresponds to weighting the measurements less than the dynamics model. This also leads to a reduction of the poles values for $A-L C$. The relative magnitude 
Table 2: PWPF parameters used to compose the ACS.

\begin{tabular}{lllcl}
\hline$k_{m}$ & $\tau_{m}$ & $U_{\text {on }}$ & $h$ & $K_{p}$ \\
1 & 0.1 & 0.45 & 0.3 & 20 \\
\hline
\end{tabular}

Table 3: Simulation parameters.

\begin{tabular}{lc}
\hline Parameters & Values \\
\hline & $J_{x}=305.89126$ \\
Principal momentum of inertia (without payload) $\left(\mathrm{kgm}^{2}\right)$ & $J_{y}=314.06488$ \\
& $J_{z}=167.33919$ \\
Torque arm $(\mathrm{m})$ & $l=1.0$ \\
Mean orbital motion $(\mathrm{rad} / \mathrm{s})$ & $\omega_{0}=0.001$ \\
Mass $(\mathrm{kg})$ & 578.05239 \\
Orbit altitude $(\mathrm{km})$ & 750 \\
Maximum force $(\mathrm{N})$ & 5 \\
Eccentricity & $\cong 0$ \\
Initial attitude (degrees) slew maneuver & $(\phi, \theta, \psi)=(10,10,10)$ \\
Initial Angular Rate $($ degrees/s) & $\boldsymbol{\omega}_{i b}^{b}=[1,1,1]^{T}$ \\
\hline
\end{tabular}

of $R_{e}$ and $Q_{e}$ is determined iteratively till achieves satisfactory gain $L$ in terms of filtering and smoothing of the measurement vector signal $\mathbf{y}_{v}$. The matrices values are given by

$$
\begin{aligned}
& Q_{e}=\operatorname{diag}\left(0,0,0, q_{e}, q_{e}, q_{e}\right), \\
& R_{e}=\operatorname{diag}\left(r_{e}, r_{e}, r_{e}, v_{e}, v_{e}, v_{e}\right),
\end{aligned}
$$

where $q_{e}=5 \times 10^{-3}, r_{e}=1 \times 10^{-1}$, and $v_{e}=1 \times 10^{-2}$. Note that the precision for the rate measurements is bigger than for the attitude measurements, and the tuning values for the dynamic noise in the attitude are selected as zeros.

\subsubsection{Selected PWPF Parameters}

In order to compose the entire reaction thruster attitude control system and to achieve the desire performance the parameters for the PWPF are selected from the optimal range. Table 2 presents those PWPF parameters.

Next section presents the performance of the reaction thruster attitude controller during the stabilization mode. Filtering noise, rejection of impulse disturbances, and regulation performance are analyzed.

\section{Numerical Simulation and Results}

The reaction thruster attitude control is tested through numerical simulations. The tuning matrices schemes presented in Section 3 are used to obtain the controller and observer gains. They are able to attempt pointing requirements $\left(<0.5^{\circ}\right)$ and reasonable margins of stability for the attitude control system during the stabilization mode. Table 3 includes the values of 


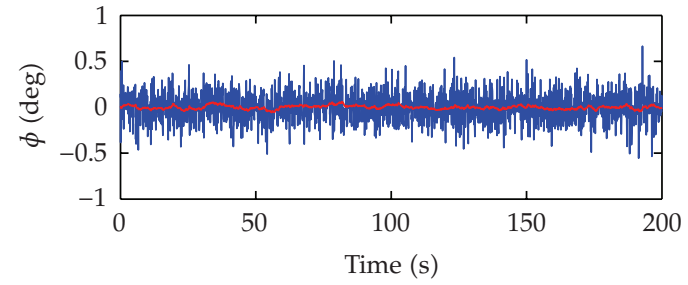

(a)

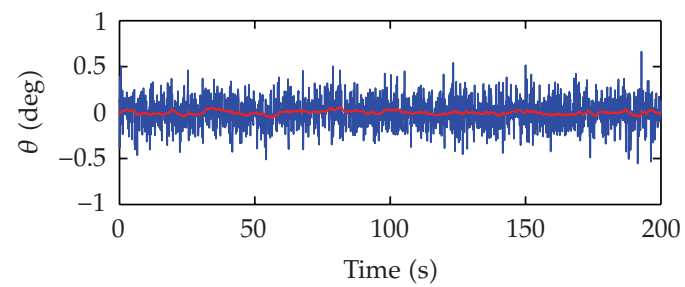

(c)

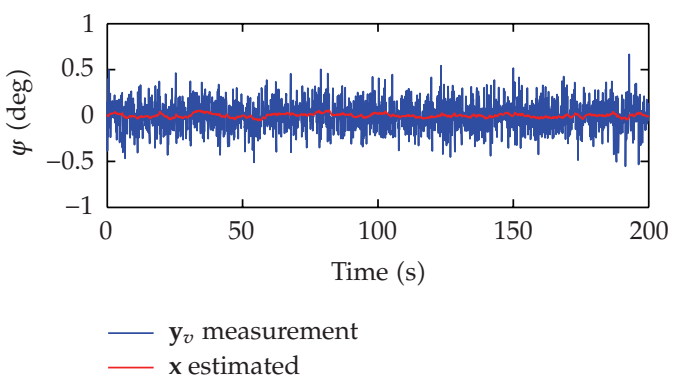

(e)

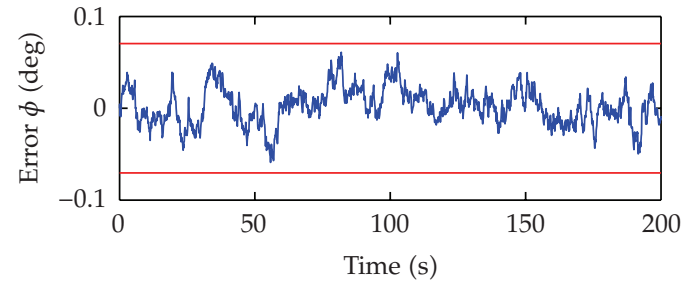

(b)

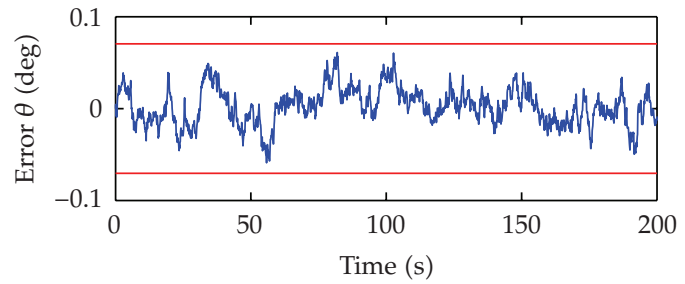

(d)

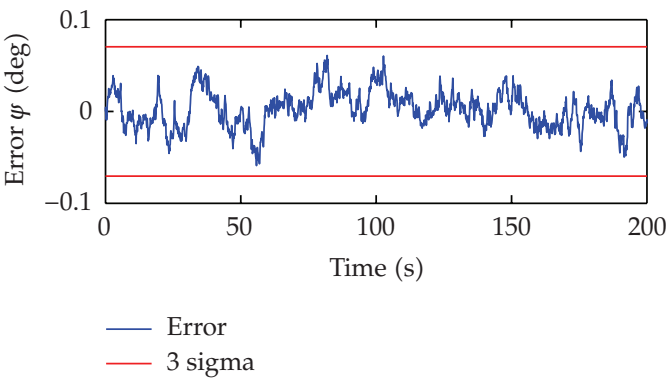

(f)

Figure 4: Filtering of the measurement data.

principal momentum of inertia without payload [1]. Although several simulations over a wide range of initial condition for attitude were performed, just one case is shown.

\subsection{Noise Filtering}

In order to filter the noise in the measurement $\mathbf{y}_{v}$ the steady-state Kalman filter is applied. The estimated and measurement attitude is shown in Figure 4, on the left side. The errors and the respective 3 sigma boundary results are shown on the right side of Figure 4 . The steady-state Kalman achieves good estimation of the real attitude with a standard deviation $\sigma \cong 0.027$. The estimation results present smoother profile compare to the measurements which is favorable wish for the control system.

\subsection{Short Slew Maneuver during Stabilization Mode}

Although a set of different initial conditions are simulated and analyzed, we present only the case when the satellite has a displacement of 10 degrees for each axis in attitude with respect to LHLV orbital frame. To regulate or stabilize the satellite a short maneuver is needed. The 


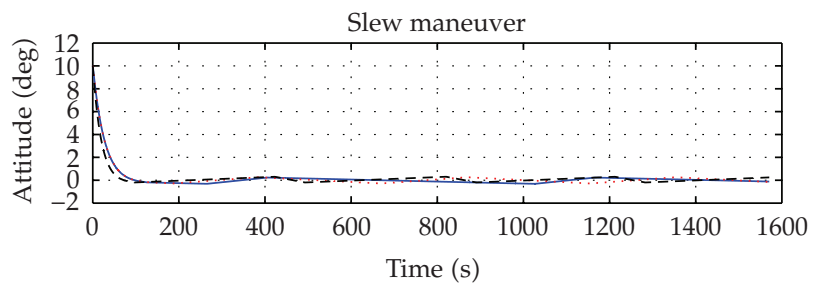

(a)

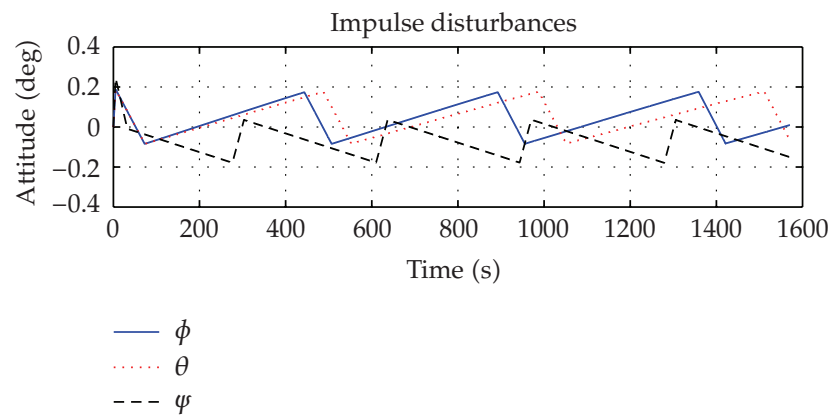

(b)

Figure 5: Attitude profile during slew maneuver and disturbance effect.

attitude profile is shown in Figure 5. The simulation time corresponds to quarter of the orbital period ( $\cong 104$ ) minutes. The satellite executes the maneuver in approximately 100 seconds.

The duty cycle generated by the PWPF modulation is shown in Figure 6. The duty cycle for row, pitch, and yaw angles are the same order of magnitude. The maximal spent time to complete a close path is quasi 800 seconds and it occurs in row direction. The specification of pointing accuracy is achieved, less than 0.5 degrees. In fact reading out Figure 6 the maximal errors in row, pitch, and yaw are $\pm 0.3, \pm 0.3$, and \pm 0.25 degrees, respectively. It shows a high accurate performance of the reaction thruster which is possible by modulating the control signal using the PWPF modulator.

Figure 7 shows the control command, executed by LQG controller, and the modulation during the slew maneuver. The thrusters' profile present small pulse-width modulation $\left(t_{\text {on }}\right)$ which leads small impulses and hence less fuel consumption. Positive torques are executed by 3 of thrusters and negative ones by another 3 thrusters.

\subsection{Disturbance Rejection During Stabilization Mode}

In order to test the action of the controller against disturbance effects (e.g., atmospheric drag) or uncertainties in the system (e.g., sloshing), simulations considering impulse disturbance signals acting in the system are carried out. Figure 5 presents the results obtained for the attitude. It shows a maximal error in attitude of \pm 0.2 degrees which fulfill the desired precision specification. The attitude control system is capable of respond fast to the disturbance effects, less than 60 seconds for an exogenous pulse of 1 Newton. The control signal command and the PWPF modulation results are presented in Figure 8. The results are satisfactory in terms of accuracy and fuel since the modulation of the pulse-width is kept small. 

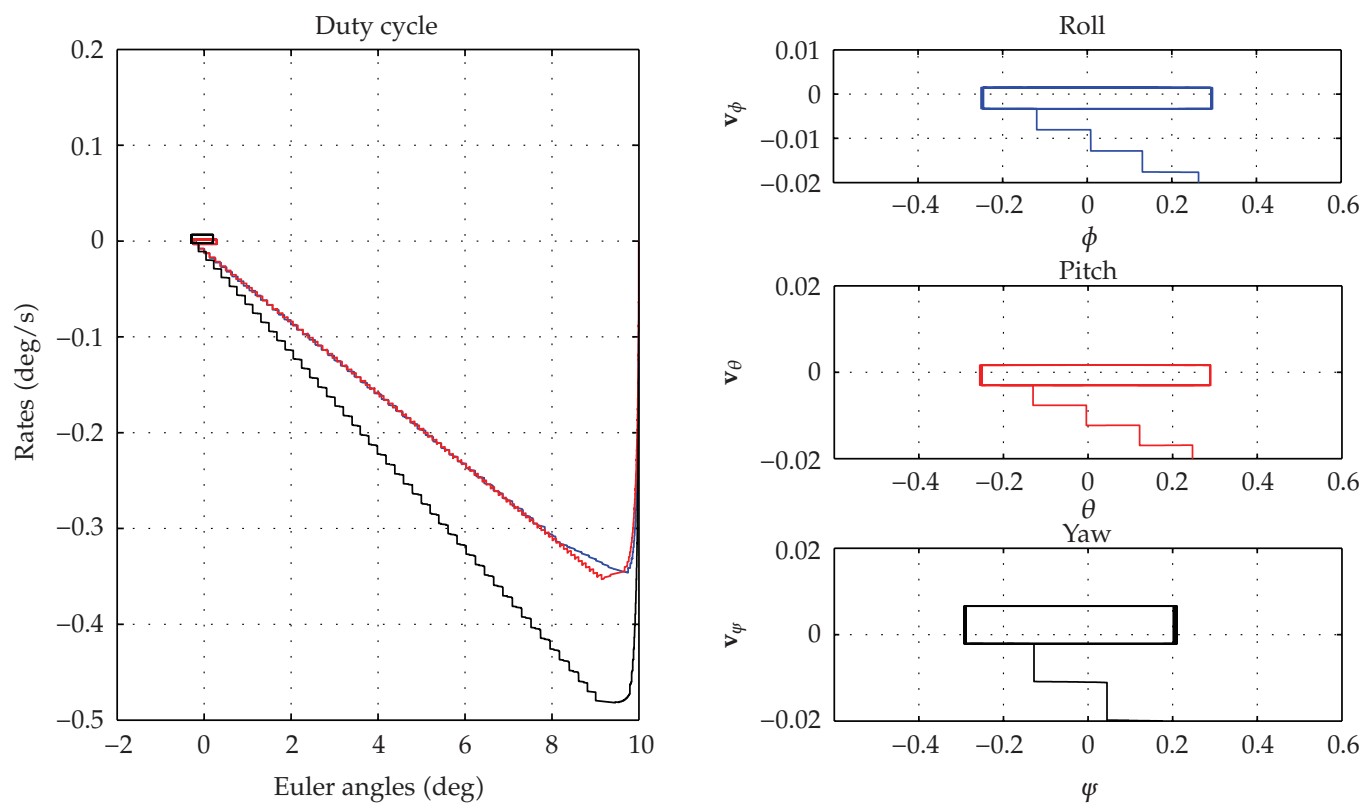

Figure 6: Duty cycle response for stabilization during a short slew maneuver.
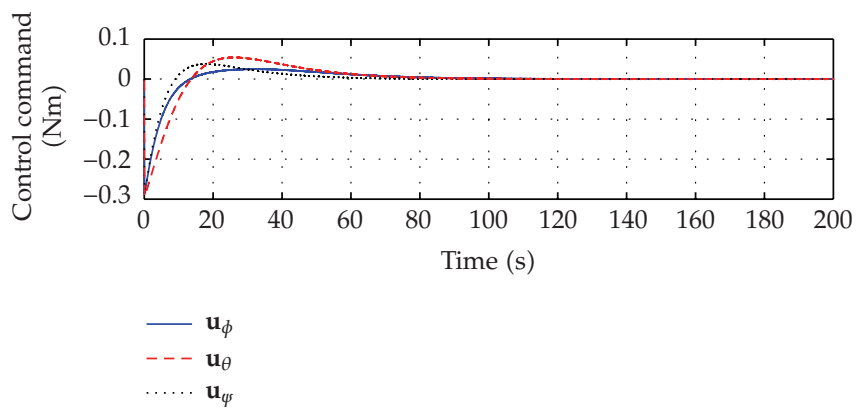

(a)

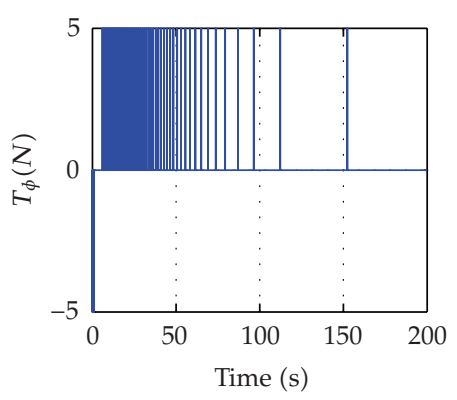

(b)

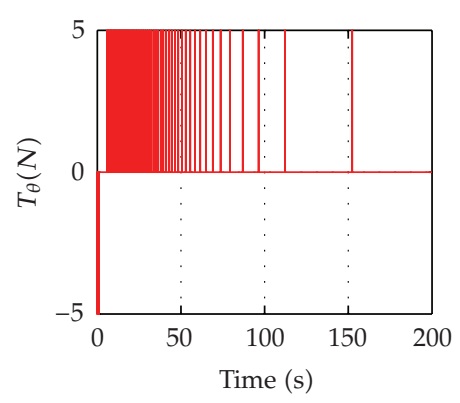

(c)

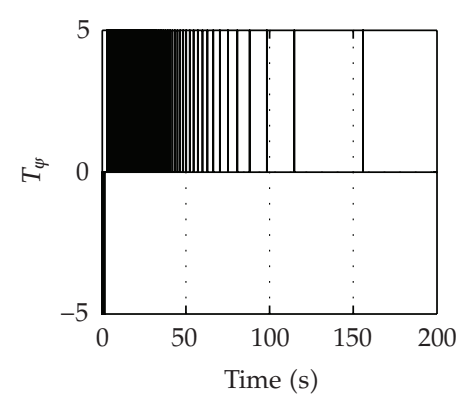

(d)

Figure 7: Control command and PWPF modulation during the slew maneuver. 


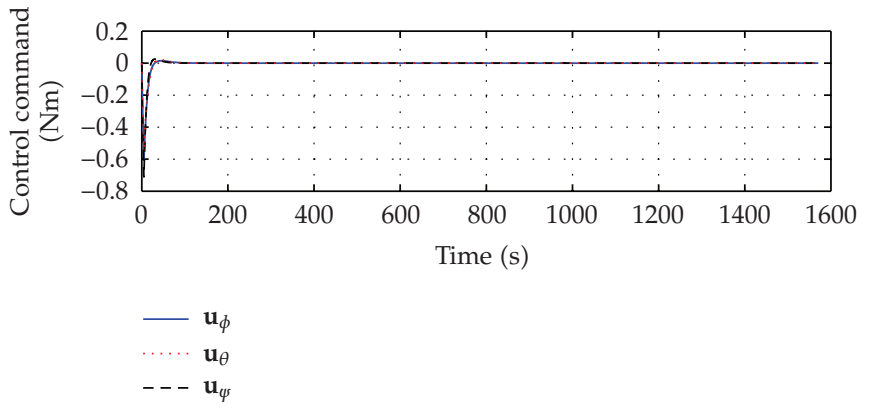

(a)

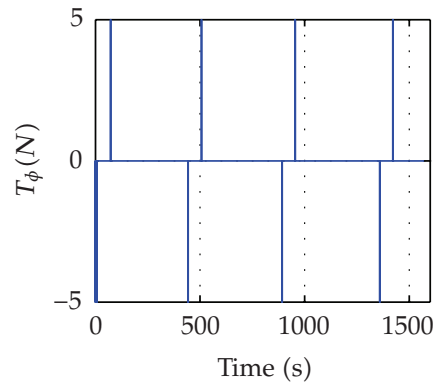

(b)

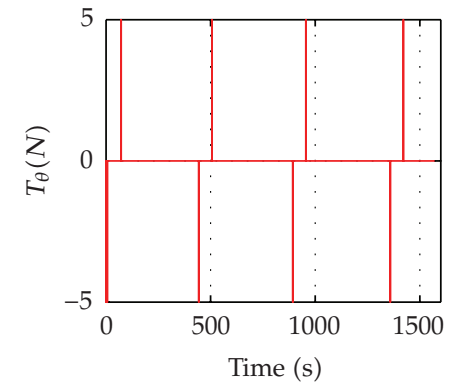

(c)

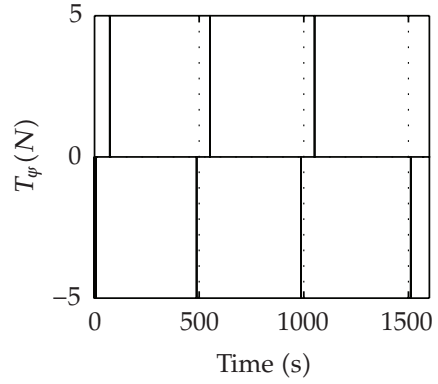

(d)

Figure 8: Control command and PWPF modulation for disturbance rejection.

It is worth to note that the use of magnetic coils actuators or reaction wheel devices can zero the error residue, for example, duty cycle, by a damped actuation. This actuation will be very small because of the level of accuracy in attitude achieved by the thruster actuation.

\section{Conclusions}

The obtained on-off thruster reaction attitude control system based on the LQG/PWPF modulation is optimal with respect to regulation, (i.e., minimizing the quadratic cost function of states and control signals), and propellant consumption. The optimality for fuel is obtained through off-line simulations varying the parameters of the PWPF modulator till less fuel consumption is achieved. This work presents the set of optimal parameters for the PWPF modulator by considering static and dynamic analysis.

The LQG design is an efficient way to achieve exponentially stability, moreover it allows to weight the magnitude of input signal $\mathbf{u}$, restricting the torque commands till acceptable performance is achieved. The weighting matrices for tuning the optimal LQG controller are presented and discussed in this work. The previous work, see [6], using the LQG design, demonstrated successfully, the applicability and suitability of the controller for the stabilization mode. However, in the foregoing work the required on-off modulation was not taken into consideration. It is worth to note that the LQG controller is able to stabilize the system even for large initial attitude displacements within nonlinear dynamics. It shows how resistent the controller is in face of internal changes in behavior.

The obtained results demonstrate the feasibility of combining LQG/PWPF modulator in an unique controller for on-off thruster reaction attitude control system. Stability remains 
by adding the PWPF modulator and reasonable accuracy in attitude is achieved, that is, magnitude of the duty cycle. Practical aspects are included in this study as filtering and presence of external impulsive perturbations. The advantages of less spent propellant shall contribute to the MMP project, specially, a satellite conceived to be used on a large number and different types of missions, in the context of an ever-advancing Brazilian space program.

\section{Acknowledgments}

The authors acknowledge the support of Conselho Nacional de Desenvolvimento Cientifico e Tecnológico-CNPq, Coordenação de Aperfeiçoamento de Pessoal de Nivel Superior CAPES (Brazil), and Fundação de Amparo à Pesquisa do Estado de S. Paulo-FAPESP (São Paulo, Brazil).

\section{References}

[1] AEB-Agência Espacial Brasileira, "National space activities program PNAE 1998-2007," Report Brazilian Space Agency, AEB, Brasilia, Brazil, 1998.

[2] Q. Hu and Y. Liu, "A hybrid scheme of feed-forward/feedback control for vibration suppression of flexible spacecraft with on-off actuators during attitude maneuver," International Journal of Information Technology, vol. 11, no. 12, pp. 95-107, 2005.

[3] T. D. Krovel, Optimal tunning of PWPF modulator for attitude control, M.S. thesis, Department of Engineering Cybernetics, Norwegian University of Science and Technology, 2005.

[4] T. D. Krovel, "PWPF modulation of thrusters on the micro-satellite SSETI/ESMO," Tech. Rep., Department of Engineering Cybernetics, Norwegian University of Science and Technology, 2005.

[5] R. S. McClelland, Spacecraft attitude control system performance using pulse-width pulse-frequency modulated thrusters, M.S. thesis, Naval Postgraduate School, Monterey, Calif, USA, 1994.

[6] A. C. Santana, L. S. Martins-Filho, and G. Arantes Jr., "Attitude stabilization of the PMM satellite using a LQG-based control strategy," Tendências em Matemática Aplicada e Computacional, vol. 9, no. 2, pp. 321-330, 2008.

[7] J. R. Wertz, Ed., Spacecraft Attitude Determination and Control, Reidel, Dordrecht, The Netherlands, 1978.

[8] M. H. Kaplan, Modern Spacecraft Dynamics and Control, John Wiley \& Sons, New York, NY, USA, 1976.

[9] B. Wie, H. Weiss, and A. Arapostathis, "Quaternion feedback regulator for spacecraft eigenaxis rotations," Journal of Guidance, Control, and Dynamics, vol. 12, no. 3, pp. 375-380, 1989.

[10] G. Arantes Jr., Estudo comparativo de técnicas de controle de atitude em três eixos para satelites artificias, M.S. thesis, INPE, S. José dos Campos, Brazil, 2005.

[11] LIGOS, “V-Relam Builder: User's Guide and Reference," Ligos Corporation, 1997.

[12] J. B. Burl, Linear Optimal Control, Prentice Hall, Upper Saddle River, NJ, USA, 1998.

[13] B. D. O. Anderson and J. B. Moore, Optimal Control: Linear Quadratic Methods, Dover, Mineola, Tex, USA, 2007. 


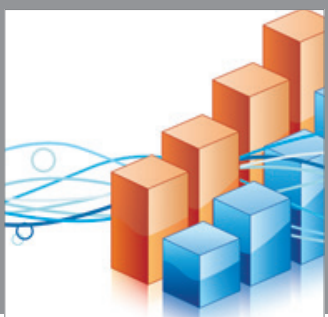

Advances in

Operations Research

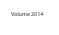

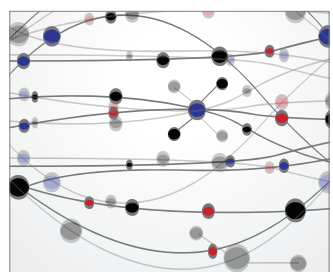

\section{The Scientific} World Journal
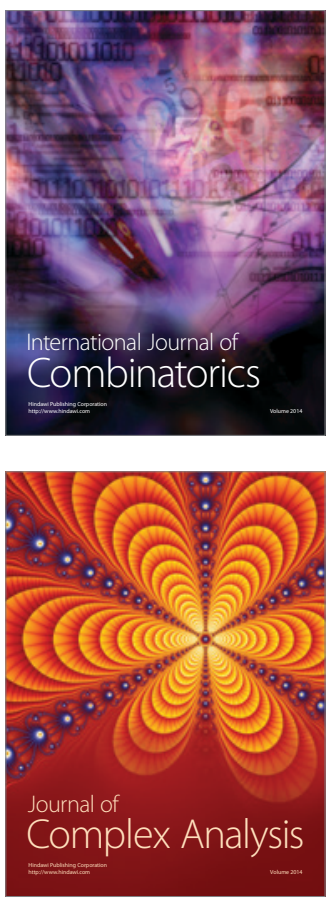

International Journal of

Mathematics and

Mathematical

Sciences
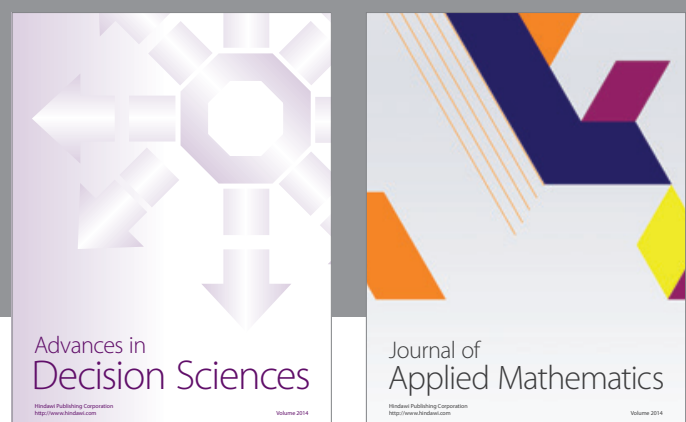

Journal of

Applied Mathematics
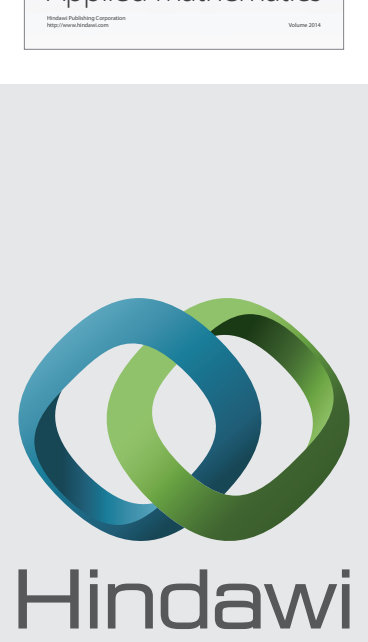

Submit your manuscripts at http://www.hindawi.com
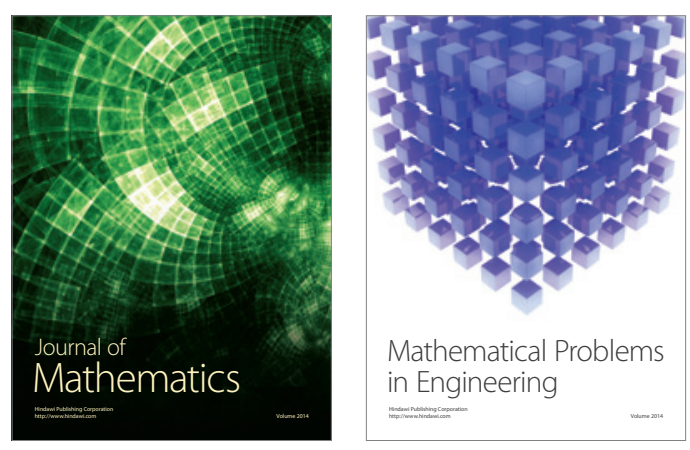

Mathematical Problems in Engineering
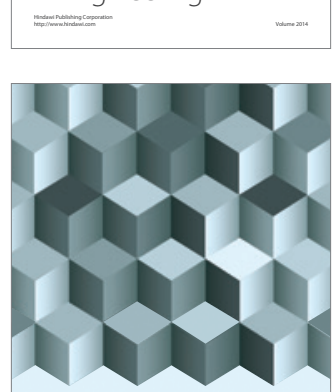

Journal of

Function Spaces
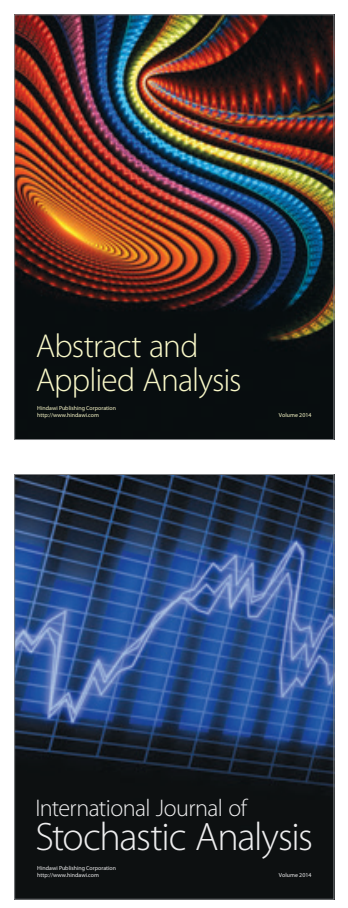

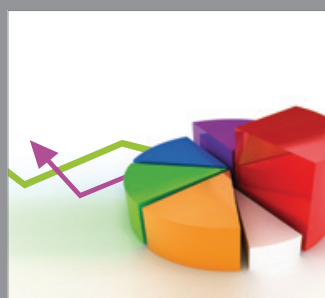

ournal of

Probability and Statistics

Promensencen
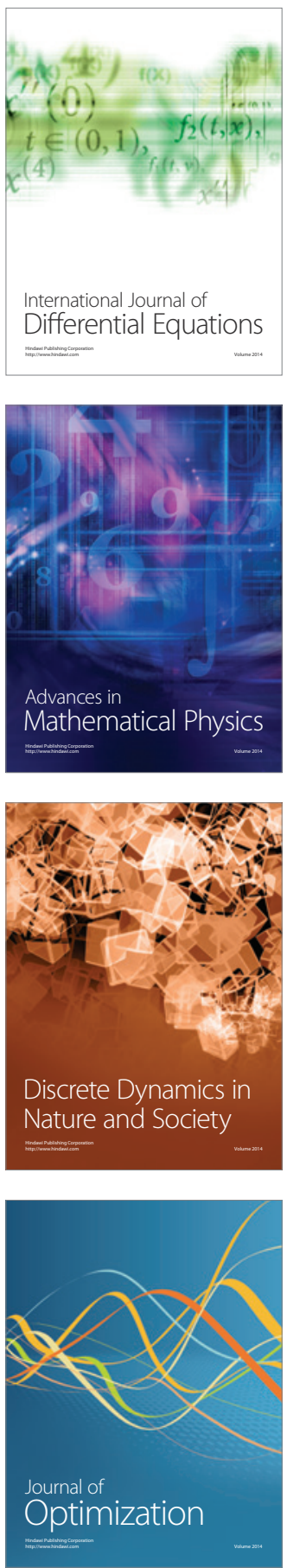